\title{
Samples of geometric random variables with multiplicity constraints
}

\author{
Margaret Archibald and Arnold Knopfmacher. \\ The John Knopfmacher Centre for Applicable Analysis and Number Theory, Department of Mathematics, \\ University of the Witwatersrand, P. O. Wits, 2050, Johannesburg, South Africa \\ marchibaldemaths.wits.ac.za, arnoldk@cam.wits.ac.za
}

We investigate the probability that a sample $\Gamma=\left(\Gamma_{1}, \Gamma_{2}, \ldots, \Gamma_{n}\right)$ of independent, identically distributed random variables with a geometric distribution has no elements occurring exactly $j$ times, where $j$ belongs to a specified finite 'forbidden set' $A$ of multiplicities. Specific choices of the set $A$ enable one to determine the asymptotic probabilities that such a sample has no variable occuring with multiplicity $b$, or which has all multiplicities greater than $b$, for any fixed integer $b \geq 1$.

Keywords: Geometric random variable, Mellin transform, Poisson transform, multiplicity

\section{Introduction}

We study samples of independent, identically distributed (i.i.d.) random variables with a geometric distribution. Specifically, let $\Gamma_{1}, \Gamma_{2}, \Gamma_{3}, \ldots$ be i.i.d. geometric random variables with parameter $p$, that is, $\mathbb{P}\left(\Gamma_{1}=j\right)=p q^{j-1}, j \in \mathbb{N}$, with $p+q=1$. There is now an extensive literature on the combinatorics of geometric random variables and its applications in Computer Science. We are interested in the probability that a random sample of $n$ such variables consists of elements whose multiplicities belong to specified sets. That is, we place restrictions on the number of times any element/letter can occur in the sample.

As a simple example, we may wish to consider a sample where none of the $n$ elements occur exactly $b$ times. In this case $A=\{b\}$. Another example of such a forbidden set is when a letter can occur only $b$ times or more (or not at all), i.e., $A=\{1,2, \ldots, b-1\}$, where $b \geq 2$. Note that we do not allow 0 in the forbidden set. Previously in (HK05, LP05), certain geometric samples with 0 in the forbidden set were studied under the names of 'complete' and 'gap-free' samples.

Theorem 1 Let $A$ be any finite set of positive integers. The probability $p_{n}$ that a geometric sample of length $n$ has no letter appearing with multiplicity $j$, for any $j \in A$ is (asymptotically as $n \rightarrow \infty$ ),

$$
p_{n}=1-\frac{T^{*}(0)}{\ln (1 / q)}-\frac{2}{\ln (1 / q)} \Re\left(\sum_{k=1}^{\infty} \exp \left\{\chi_{k} \ln (q / n)\right\} T^{*}\left(\chi_{k}\right)\right)+O\left(n^{-1}\right),
$$

where we set $\chi_{k}:=\frac{2 k \pi i}{\ln (1 / q)}$, and where

$$
T^{*}(0)=\sum_{j \in A} p^{j} \sum_{n \geq 0} p_{n} q^{n} \frac{1}{n+j}\left(\begin{array}{c}
n+j \\
j
\end{array}\right)
$$

and

$$
T^{*}\left(\chi_{k}\right)=\sum_{j \in A} \frac{p^{j}}{j !} \sum_{n \geq 0} p_{n} \frac{q^{n}}{n !} \Gamma\left(n+j+\chi_{k}\right), \quad \text { for } \quad k \in \mathbb{Z} \backslash\{0\} .
$$

${ }^{\dagger}$ This material is based upon work supported by the National Research Foundation under grant number 2053740 


\section{Outline of the proof of Theorem 1}

We want a recursion on the variable $p_{n}$, the probability that the sample does not have letters which appear exactly $j$ times if $j$ is an element of the forbidden set $A \subset \mathbb{N}$. Let $\mathcal{B}$ represent the set of all permitted samples. If we let $p_{n}=\mathbb{P}(\Gamma \in \mathcal{B})$ (the probability that the sample $\Gamma=\left(\Gamma_{1}, \Gamma_{2}, \ldots, \Gamma_{n}\right)$ has no letter occurring exactly $j$ times for $j \in A$ ), then we can write

$$
p_{n}=\sum_{\substack{j \geq 0 \\ j \notin A}} \mathbb{P}\left(\{\Gamma \in \mathcal{B}\} \cap\left\{\sum_{\ell=1}^{n} I_{\Gamma_{\ell}=1}=j\right\}\right)=\sum_{\substack{j \geq 0 \\ j \notin A}} \mathbb{P}\left(\Gamma \in \mathcal{B} \mid \sum_{\ell=1}^{n} I_{\Gamma_{\ell}=1}=j\right) \cdot \mathbb{P}\left(\sum_{\ell=1}^{n} I_{\Gamma_{\ell}=1}=j\right),
$$

where the indicator function $I$ takes values 1 for true and 0 for false. Using the law of total probability, and the memoryless property of geometric random variables, we obtain the recursion:

$$
p_{n}=\sum_{j=0}^{n} p_{n-j}\left(\begin{array}{l}
n \\
j
\end{array}\right) p^{j} q^{n-j}-\sum_{j \in A} p_{n-j}\left(\begin{array}{l}
n \\
j
\end{array}\right) p^{j} q^{n-j}
$$

We would like to see how $p_{n}$ behaves asymptotically as $n \rightarrow \infty$. The Poissonisation technique we use can be seen in (JS98; Szp01), but we follow more specifically the process used in (HK05; JS97). Namely, we consider the Poisson transform of the sequence $\left(p_{n}\right)$, analyse its asymptotics with Mellin transforms, then de-Poissonise to recover the asymptotics of $\left(p_{n}\right)$. To do this, we make use of the exponential generating function $P(z)$, which is the Poisson transform of $\left(p_{n}\right)$, given by $P(z):=\sum_{n \geq 0} p_{n} \frac{z^{n}}{n !} e^{-z}$. We use 2.1 to show that

$$
P(z)-e^{-z}=\sum_{n \geq 1} p_{n} \frac{z^{n}}{n !} e^{-z}=P(q z)-e^{-p z} \sum_{j \in A} \frac{(p z)^{j}}{j !} P(q z)-e^{-z} .
$$

We thus have a functional equation of the form:

$$
P(z)=P(q z)-e^{-p z} \sum_{j \in A} \frac{(p z)^{j}}{j !} P(q z) .
$$

The technique we now use is the Mellin transform. A standard reference on Mellin transforms is (FGD95). We define the function (see 2.2)

$$
T(z):=e^{-p z} \sum_{j \in A} \frac{(p z)^{j}}{j !} P(q z) \quad\left(=\sum_{n \geq 0} p_{n} \frac{q^{n}}{n !} \sum_{j \in A} \frac{p^{j}}{j !} z^{n+j} e^{-z}\right) .
$$

We note that the Mellin transform of $T(z)$ has a fundamental strip of at least $\langle-1, \infty\rangle$. Now we find the Mellin transform of 2.3 to be

$$
T^{*}(s)=\sum_{n \geq 0} p_{n} \frac{q^{n}}{n !} \sum_{j \in A} \frac{p^{j}}{j !} \mathcal{M}\left(z^{n+j} e^{-z}\right)=\sum_{n \geq 0} p_{n} \frac{q^{n}}{n !} \sum_{j \in A} \frac{p^{j}}{j !} \Gamma(n+j+s) .
$$

In particular we will make use of the values $T^{*}(0)$ and $T^{*}\left(\chi_{k}\right)$, as given in 1.1 and 1.2 . After iterating (2.2) we get

$$
P(z)=P(q z)-T(z)=P\left(q^{m+1} z\right)-\sum_{j=0}^{m} T\left(q^{j} z\right),
$$

for any $m \geq 0$ and thus in the limit as $m \rightarrow \infty, P(z)=1-\sum_{j=0}^{\infty} T\left(q^{j} z\right)$. We define $Q(z):=P(z)-1=$ $-\sum_{j=0}^{\infty} T\left(q^{j} z\right)$. Then the corresponding Mellin transform is

$$
Q^{*}(s)=-\sum_{j=0}^{\infty} q^{-j s} T^{*}(s)=-\frac{T^{*}(s)}{1-q^{-s}}=\frac{T^{*}(s)}{q^{-s}-1},
$$

where $Q^{*}(s)$ exists in the fundamental strip $\langle-1,0\rangle$. The inverse Mellin transform is

$$
Q(x)=\frac{1}{2 \pi i} \int_{c-i \infty}^{c+i \infty} Q^{*}(s) x^{-s} d s
$$


for any $-1<c<0$. The residue theorem can be used to evaluate this. This process (compare to ([HK05) results in

$$
Q(x) \sim-\sum_{\chi} \operatorname{Res}_{s=\chi}\left(Q^{*}(s) x^{-s}\right) .
$$

Now, $x^{-s} Q^{*}(s)=x^{-s} T^{*}(s) /\left(q^{-s}-1\right)$ has simple poles at $q^{-s}=1$, i.e. at $\chi_{k}=2 k \pi i / \ln (1 / q), k \in \mathbb{Z}$, and the corresponding residues are

$$
\lim _{s \rightarrow \chi_{k}}\left(s-\chi_{k}\right) \frac{x^{-s} T^{*}(s)}{q^{-s}-1}=\frac{x^{-\chi_{k}} T^{*}\left(\chi_{k}\right)}{q^{-\chi_{k}} \ln (1 / q)}=\left(\frac{q}{x}\right)^{\chi_{k}} \frac{T^{*}\left(\chi_{k}\right)}{\ln (1 / q)} .
$$

Of these quantities, all but the $k=0$ term contribute oscillations of small amplitude. We need to use asymptotic de-Poissonisation to deduce that $p_{n} \sim P(n)=Q(n)+1$. We consider the theorem given in Szpankowski, (Szp01, page 463), whose five conditions are met by choosing $\gamma_{1}(z)=0, \gamma_{2}(z)=1$, and $t(z)=-T(z)$. We can now deduce that

$$
p_{n}=P(n)+O\left(n^{-1}\right)=Q(n)+1+O\left(n^{-1}\right)
$$

where

$$
P(n)=Q(n)+1 \sim 1-\frac{T^{*}(0)}{\ln (1 / q)}-\frac{2}{\ln (1 / q)} \Re\left(\sum_{k=1}^{\infty} \exp \left\{\chi_{k} \ln (q / n)\right\} T^{*}\left(\chi_{k}\right)\right),
$$

with $T^{*}(0)$ and $T^{*}\left(\chi_{k}\right)$ given in 1.1 and 1.2 respectively. This concludes the proof of Theorem 1 .

\section{The complementary set $\mathbb{N} \backslash A$ is finite}

We consider now the complementary problem where the permitted set $B=\mathbb{N} \backslash A$ is finite. The dePoissonisation method cannot be used in this case, but we can bound the probabilities by elementary means to show that $p_{n} \rightarrow 0$ for all finite sets $B$. (Consequently fluctuations are also absent in these cases.) Suppose the permitted set of multiplicities $B=\mathbb{N} \backslash A$ is finite with largest element $k$. The probability that all multiplicities belong to such a set $B$ is bounded above by the case when $B=\{0,1,2, \ldots, k\}$. Now samples where all multiplicities are at most $k$ are themselves a subset of the set of samples with the weaker restriction that there are at most $k$ ones.

The probability of exactly $j$ ones in a sample of length $n$ is $\left(\begin{array}{c}n \\ j\end{array}\right) p^{j}(1-p)^{n-j}$. Hence the probability of at most $\mathrm{k}$ ones is

$$
\sum_{j=0}^{k}\left(\begin{array}{l}
n \\
j
\end{array}\right) p^{j}(1-p)^{n-j} \leq(k+1)\left(\begin{array}{l}
n \\
k
\end{array}\right)(1-p)^{n-k}=O\left(n^{k} q^{n}\right),
$$

which is exponentially small.

\section{Further work}

We aim to continue this work by considering other examples of forbidden sets $A$. In addition, we would like to reconsider the results of this paper from an urn model standpoint, as was used to obtain the results in (LP05).

\section{Acknowledgements}

We would like to thank Helmut Prodinger for the helpful advice he gave at the start of this project, relating to finding asymptotics from the recursion 2.1 .

\section{References}

[FGD95] P. Flajolet, X. Gourdon, and P. Dumas. Mellin transforms and asymptotics: Harmonic sums. Theoretical Computer Science, 144:3-58, 1995.

[HK05] P. Hitczenko and A. Knopfmacher. Gap-free compositions and gap-free samples of geometric random variables. Discrete Mathematics, 294:225-239, 2005.

[JS97] S. Janson and W. Szpankowski. Analysis of the asymmetric leader election algorithm. Electr. J. Combin., 4(1, research paper 17):16pp, 1997. 
[JS98] P. Jacquet and W. Szpankowski. Analytical de-poissonization and its applications. Theoret. Comput. Sci., 201:1-62, 1998.

[LP05] G. Louchard and H. Prodinger. The number of gaps in sequences of geometrically distributed random variables. Preprint, 304, 2005.

[Pro93] H. Prodinger. Combinatorial problems of geometrically distributed random variables and applications in computer science. Publications de l'IRMA (Strassbourg), 30:87-95, 1993.

[Szp01] W. Szpankowski. Average Case Analysis of Algorithms on Sequences. John Wiley and Sons, New York, 2001. 